OPEN ACCESS

Edited by:

Miguel Alfedo Castellan, University of Miami, United States

Reviewed by: Bashir Ahmed Qureshi, Sindh Institute of Urology and

Transplantation, Pakistan Yuval Bar-Yosef Dana-Dwek Children's Hospital, Israe

*Correspondence: Nina Younsi nina.younsi@umm.de

Specialty section: This article was submitted to Pediatric Urology, a section of the journal

Frontiers in Pediatrics

Received: 30 November 2020 Accepted: 22 March 2021 Published: 14 April 2021

Citation: Jank M, Stein $R$ and Younsi N (2021) Postnatal Management in Congenital Lower Urinary Tract Obstruction With and Without Prenatal Vesicoamniotic

Shunt. Front. Pediatr. 9:635950. doi: 10.3389/fped.2021.635950

\section{Postnatal Management in Congenital Lower Urinary Tract Obstruction With and Without Prenatal Vesicoamniotic Shunt}

\author{
Marietta Jank ${ }^{1,2}$, Raimund Stein ${ }^{1}$ and Nina Younsi ${ }^{1 *}$ \\ ${ }^{1}$ Center for Pediatric, Adolescent and Reconstructive Urology, Medical Faculty Mannheim, University of Medical Center \\ Mannheim, Heidelberg University, Heidelberg, Germany, ${ }^{2}$ Department of Pediatric Surgery, Medical Faculty Mannheim, \\ University of Medical Center Mannheim, Heidelberg University, Heidelberg, Germany
}

Purpose: Congenital lower urinary tract obstruction (cLUTO) includes a heterogeneous group of conditions caused by a functional or mechanical outlet obstruction. Early vesicoamniotic shunting (VAS) possibly reduces the burden of renal impairment. Postpartum, pediatric urologists are confronted with neonates who have a shunt in place and a potentially impassable urethra with a narrow caliber. Early management of these patients can be challenging. Here, we would like to share the approach we have developed over time.

Materials and Methods: We conducted a single-center retrospective analysis from 2016 to 2020 and included all patients diagnosed with cLUTO. Data focusing on time point and type of intervention was collected. Furthermore, patients with temporary diversion via a percutaneous VAS were selected for a more detailed review.

Results: In total, 71 cases of cLUTO were identified during the study period. Within this group, 31 neonates received postnatal management and surgical intervention in our center. VAS was performed in $55 \%$ of these cases $(N=17)$. The postnatal treatment varied between transurethral or suprapubic catheterization and early Blocksom vesicostomy. In five infants with VAS, the urinary drainage was secured through the existing VAS by inserting a gastric tube $(N=1)$ or a $4.8 \mathrm{Fr} J J$-stent $(N=4)$. To our knowledge, this is the first report of a stent-in-stent scheme, which can remain indwelling until the definite treatment.

Conclusion: Having a secure urine drainage through a VAS allows the often premature infant to grow until definite surgery can be performed. This avoids placing a vesicostomy, which requires anesthesia.

Keywords: cLUTO, lower urinary tract obstruction, fetal therapy, vesicoamniotic shunt, surgical strategies 


\section{INTRODUCTION}

Congenital lower urinary tract obstruction (cLUTO) is an obstructive uropathy that occurs in 3.3/10.000 births and predominantly affects male newborns (1). The most common underlying condition is "classical" posterior urethral valves (PUV), accounting for $\sim 56-63 \%$ of the cases (1-4). Others include anterior urethral valves, obstructive ureterocele, as well as urethral hypoplasia, stenosis and agenesis. Due to the outlet obstruction, severe oligo- or anhydramnios can occur resulting in pulmonary hypoplasia and potentially neonatal death. Of those who survive, up to a third need renal replacement therapy (5-7).

The diagnosis is mainly based on antenatal ultrasound examination. Characteristic findings include bilateral hydronephrosis with megacystis +/- thick-walled bladder. Fetal megacystis refers to a bladder diameter of $\geq 7 \mathrm{~mm}$ in the first trimester. In the second trimester, it is defined as an abnormal distension of the thick-walled bladder failing to empty within $45 \mathrm{~min}$ of observation However, half the cases of megacystis show spontaneous resolution of the megacystis (8).

Due to improved antenatal, ultrasound-based diagnosis, early fetal intervention - predominantly vesicoamniotic shunting (VAS) - is possible starting from the $12-13^{\text {th }}$ week of gestation. VAS bypasses the obstruction, restoring amniotic fluid volume. As a result, it seems to improve the pulmonary as well as the renal function leading to decreased perinatal morbidity and mortality.

Assuming that fetal kidneys start producing urine at the 10th week of gestation and that nephrogenesis takes place in the first and second trimester, some authors argue that early VAS (up to the 14th week of gestation) would significantly improve kidney function. However, a detection of co-existing syndromes or other severe, life-threatening malformations is extremely difficult at this early stage of pregnancy.

In this situation, precise and sustainable medical counseling of prospective parents poses a serious challenge.

Nevertheless, postpartum pediatric urologists are confronted with a newborn having one or multiple artificial shunts in place and a urethra often lacking a sufficient caliber for cystoscopy within the first weeks of life.

Moreover, the percutaneous shunt is often an insecure form of urine drainage. Currently, the Harrison fetal bladder stent and the Somatex ${ }^{\circledR}$ intrauterine shunt are most frequently used for vesicoamniotic shunting. The Harrison stent is a 5 Fr flexible catheter with a curled tip on both sides, whereas the Somatex ${ }^{\circledR}$ consists of a wire mesh and self-deploying parasols at both ends. Recently published literature showed an intrauterine dislocation rate between $45 \%$ (Somatex ${ }^{\circledR}$ shunt) and $85 \%$ (Harrison stent) (9). Postnatally, the newborn's growth and steady movement increases the risk of shunt dislocation, overgrowth and granulation. In our opinion, relying on the vesicoamniotic shunt as the only diversion is unsafe. Therefore, a safe temporary solution until definite treatment is needed. Options include transurethral catheterization or early vesicostomy. When a VAS with adequate drainage is in place, percutaneous placement of a suprapubic catheter is usually not possible or very difficult. However, placing an external tube through the shunt to drain the urine like a suprapubic catheter may be a solution.

Objective of this study is to draw the readers' attention to the current research gap on the immediate surgical postnatal management of these patients and present possible solutions and approaches. Hence, we want to share our 4 years of experience in treating patients with cLUTO with and without VAS after birth. Based on our analysis and results, we aim to facilitate and attempt to standardize postnatal care of newborns with cLUTO.

\section{MATERIALS AND METHODS}

After institutional ethical approval (Ethical Approval No. 2020 $873 \mathrm{R}$ ), we performed a retrospective analysis of patients with cLUTO treated at our tertiary care center between $1^{\text {st }}$ of January 2016 and $30^{\text {th }}$ of September 2020.

For our initial data collection, we identified male and female patients with cLUTO aged $0-18$ years using the ICD-10 code Q64.0-10 (congenital anomaly of the urinary system). The diagnoses meatal stenosis, exstrophy-epispadias complex and urachal anomalies were excluded and patients with a subvesical obstruction were determined. If primary postnatal treatment of cLUTO was carried out in our pediatric urology department, we included these patients for further analysis (Figure 1). Patients' characteristics (sex, gestational age and birth weight) as well as the underlying pathology of cLUTO were assessed. Furthermore, the type and time point of initial postnatal management (diversion by a urinary catheter, vesicostomy or through VAS) and definite treatment (endoscopic valve incision or vesicostomy due to other urethral pathologies) were evaluated. Demographic factors were described using median and range for continuous variables, as well as absolute and relative percentage frequencies for categorical variables.

\section{General Indication for Intrauterine Intervention}

Indications for antenatal VAS placement were bilateral severe hydronephrosis associated with a thick-walled megacystis and oligo- or anhydramnios. Amniotic fluid plays an essential role in fetal lung development and its absence may lead to pulmonary hypoplasia causing a life-threatening condition. According to recent findings, early fetal intervention can be considered at the end of the first trimester, if the following applies: (1) a longitudinal bladder diameter $>12 \mathrm{~mm}$, (2) a normal nuchal translucency (as a screening to detect chromosomal abnormalities), and (3) umbilical cord cysts could be ruled out (as a hint for urethral atresia) (10).

\section{Treatment Algorithm}

In our center, we follow an algorithm for postnatal management of cLUTO patients, as shown in Figure 2. The treatment depends on whether a vesicoamniotic shunt is in place or not.

In newborns without VAS, we once attempt transurethral catheterization for urinary drainage directly after birth. Routinely, we use 3-5 Fr feeding tubes or 6 (-8) Fr silicone foley catheters (without mandrins). If transurethral catheterization is not successful, suprapubic drainage is preferred until further or 


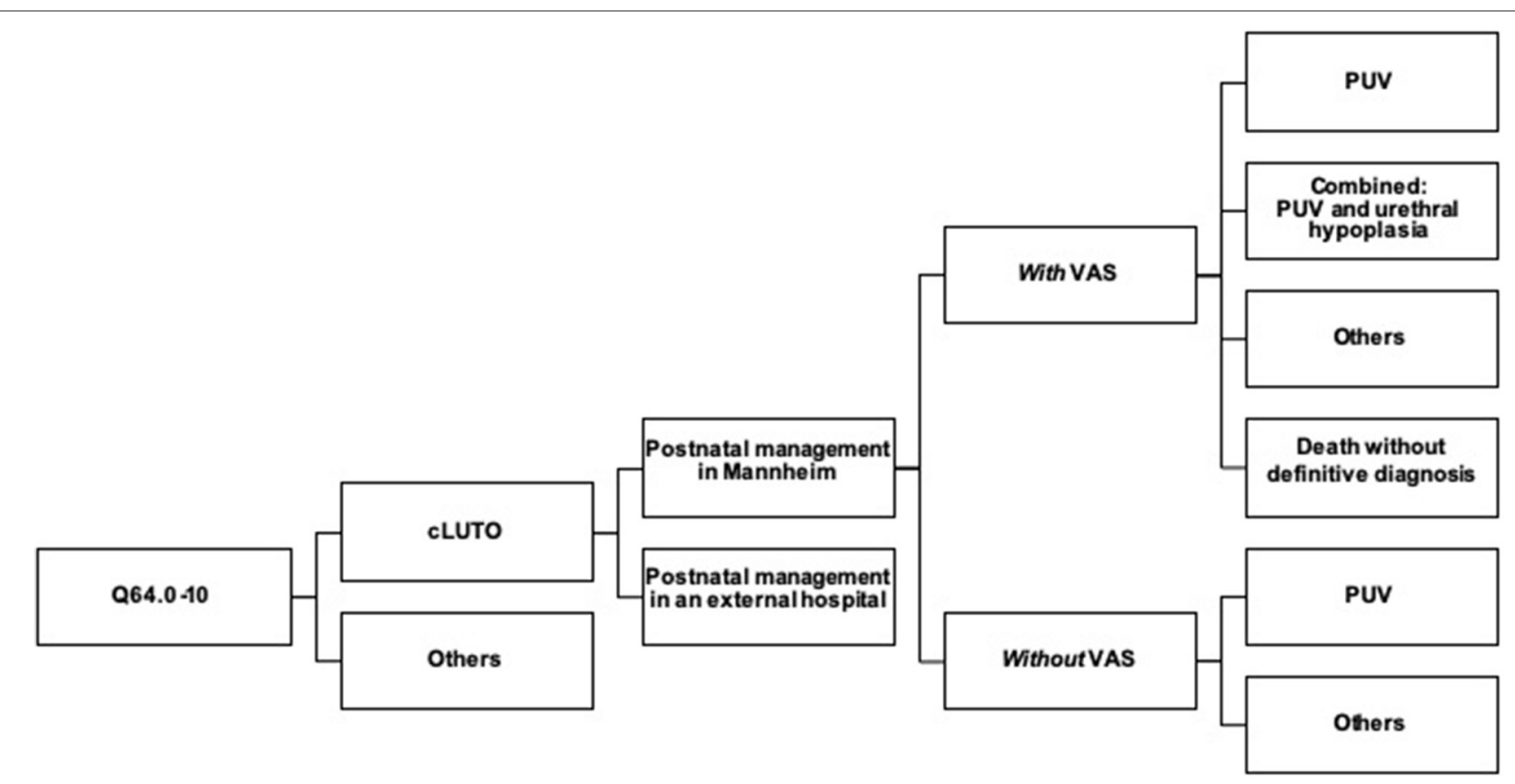

FIGURE 1 | Flowchart describing the 31 cases of CLUTO treated in our center after birth. CLUTO: congenital urinary tract obstruction, PUV: posterior urethral valves, VAS: vesicoamniotic shunting.

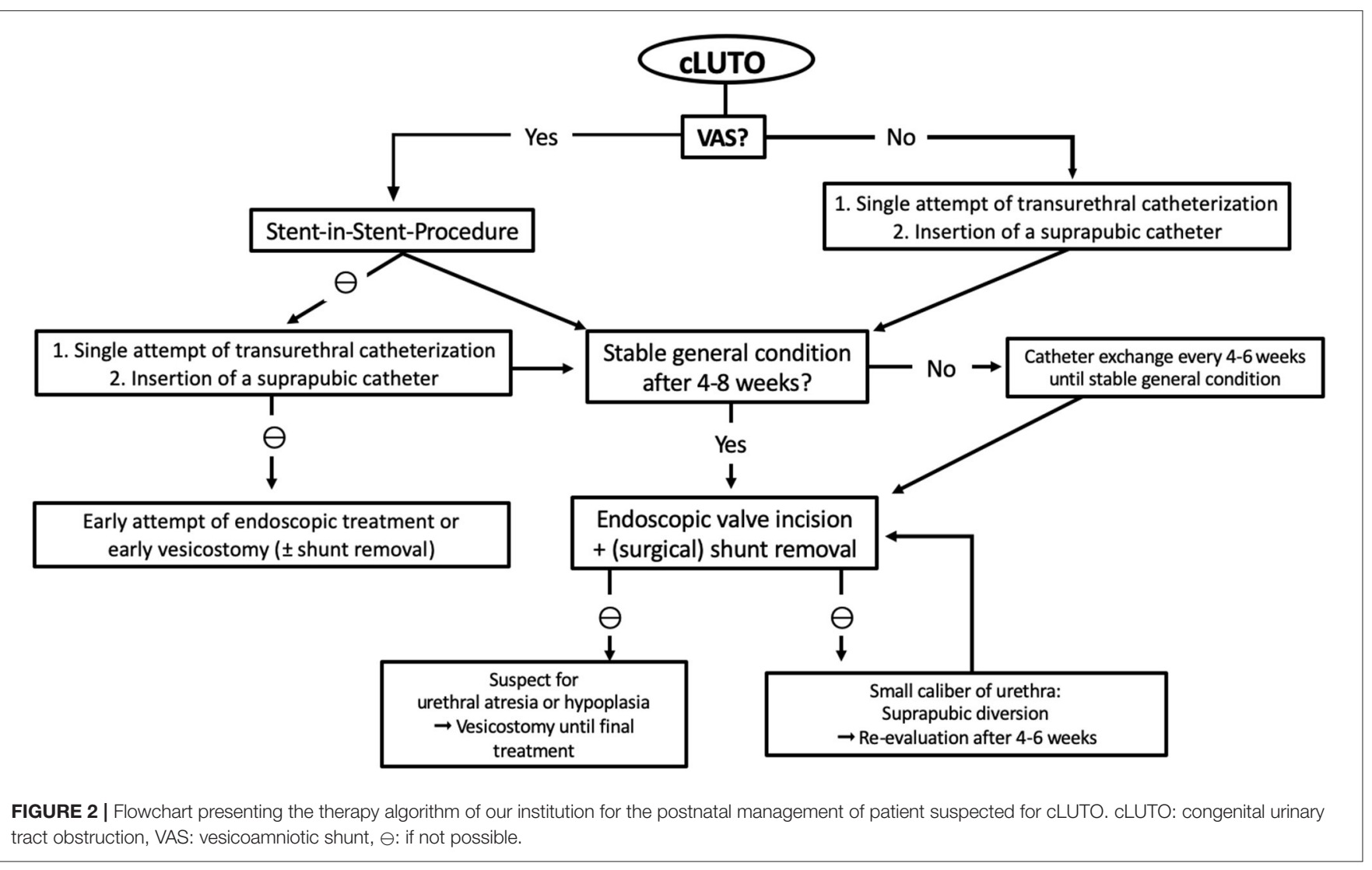


definite treatment. We use the pediatric punction set (Cystofix ${ }^{\circledR}$ ) with a 10 Fr catheter and a curled pigtail tip.

If a VAS is in place, we first recommend a stent-instent drainage. Should this procedure prove unsuccessful, we proceed as mentioned above and place a transurethral or suprapubic catheter.

The further, or even definite, therapy is determined by the newborns' general constitution and co-existing medical
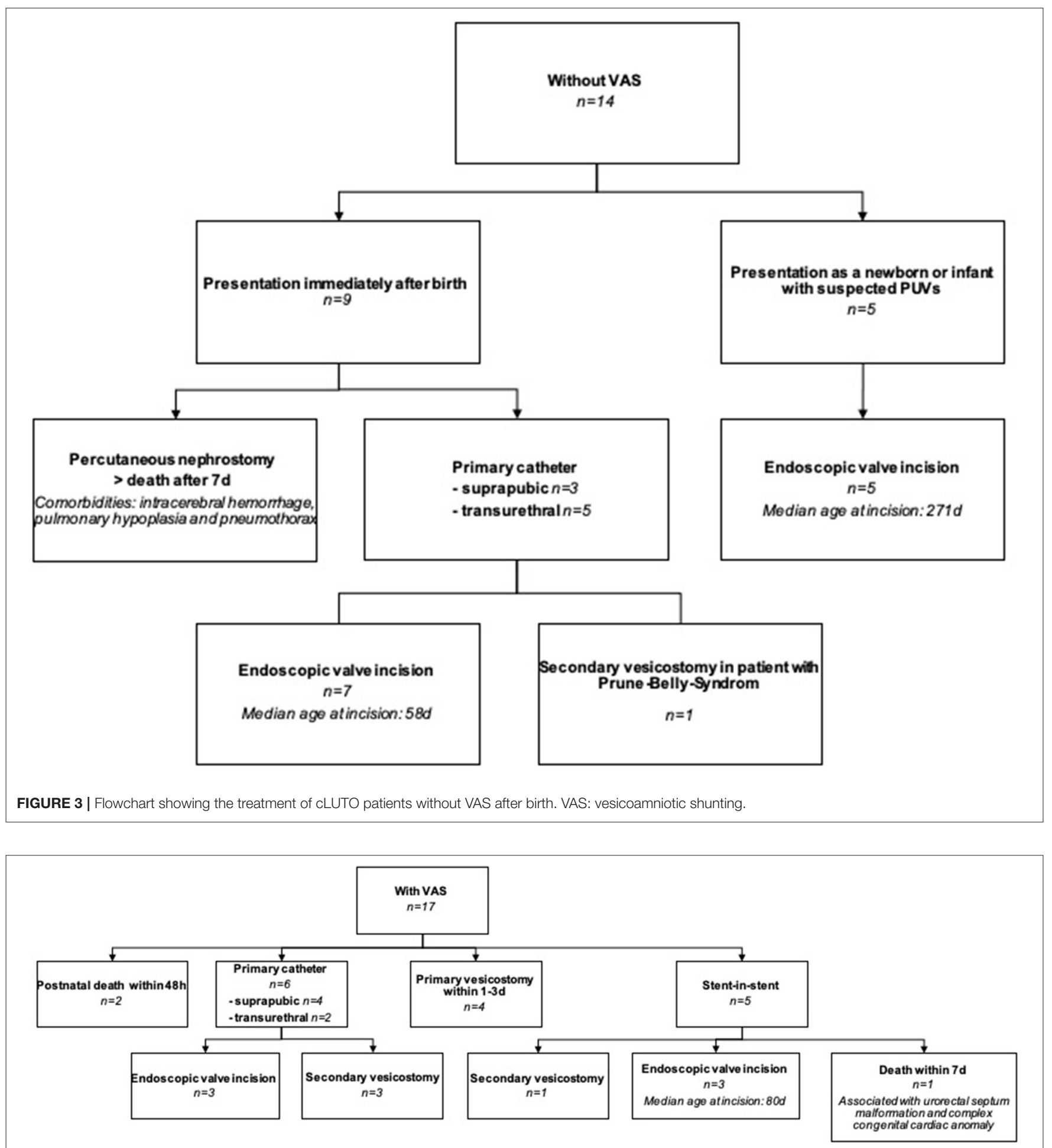

FIGURE 4 | Flowchart explaining the postnatal management of CLUTO newborns with a VAS in place. VAS: vesicoamniotic shunting. 
conditions or anomalies. If the newborn is clinically stable, an (early) urethrocystoscopy with a $6 \mathrm{Fr}$ cystoscope can be performed at 4-8 weeks of age. Our first-line procedure is the endoscopic valve incision using an $8 \mathrm{Fr}$ resectoscope with a hook-shaped cold knife. We do not use bugbee electrodes or laser fibers for urethral valve ablation to avoid strictures.

\section{RESULTS}

\section{Patient Characteristics'}

Between 2016 and 2020, a total of 209 patients with an anomaly of the lower urinary tract were treated at our tertiary care center. After exclusion of the diagnoses meatal stenosis, exstrophyepispadias complex and urachal anomalies, 71 patients with a congenital subvesical obstruction remained. There were 31 patients who received primary postnatal treatment of cLUTO in our pediatric urology department, all of which were included for further analysis.

A single patient was female and the remaining 30 patients were male. Prenatal VAS shunting was performed in 17 neonates (55\%), while 14 (45\%) had no prenatal intervention due to the diagnosis being unknown or the parents' preference for a conservative management (Figure 1).

The median week of gestation at delivery was $36^{+2}$ (range: $29^{+2}-40^{+1}$ weeks) in the VAS-group, including seven preterm neonates. On the contrary, infants without VAS were born at a median gestational age of $38^{+6}$ weeks (range: $32^{+2}-41^{+5}$ weeks). Neonates with VAS had a lower median birth weight (2,450 g, range: $1,540-3,390 \mathrm{~g})$ compared to the group without VAS (3,088 g, range: $2,710-4,160 \mathrm{~g})$.

\section{Postnatal Diagnosis}

Within the 31 infants who were postnatally managed in our center, the final diagnosis was predominantly posterior urethral valves $(N=24,77 \%)$, either isolated $(N=21,68 \%)$ or combined with a urethral hypoplasia $(N=3,10 \%)$. Other diagnoses included urethral atresia $(N=1,3 \%)$, Prune belly syndrome $(N$ $=2,6 \%)$ or an undefined anomaly $(N=1,3 \%)$. Three neonatal deaths occurred within seven days after birth (10\%). Postmortem, the parents rejected an autopsy; hence the underlying pathology remains unkown.

In the group with VAS, 8/17 infants were diagnosed with isolated posterior urethral valves and $3 / 17$ in combination with a urethral hypoplasia. Further underlying anomalies included urethral atresia $(N=1)$, Prune belly syndrome $(N=1)$ and an undefined complex urogenital-anorectal malformation in a female $(N=1)$. Furthermore, in three cases the final diagnosis remained unknown due to postnatal death. Among the 14 infants without VAS, 13 had isolated PUV and one had a Prune belly syndrome.

\section{Postnatal Management}

Whether a newborn has a VAS in situ or not, determines the postnatal treatment by the pediatric urologist. Accordingly, we compared the primary management after birth between the following two groups: cLUTO without VAS and cLUTO with VAS.

Patients without VAS were presented to our department either immediately after birth $(N=9)$ or later $(N=5)$. The following procedures were performed on the 14 cLUTO patients without VAS (Figure 3):

(1) In seven cases, a urinary catheter was placed until definite urethral valve incision: Two had a suprapubic catheter, another two had a transurethral tube and in the remaining three patients, the initial transurethral drainage was later changed to a suprapubic diversion.

(2) Early vesicostomy at an age of 17 days was performed in one newborn, after initial suprapubic urine drainage.

(3) In a single case, the neonate presented with life-limiting comorbidities (lung hypoplasia, pneumothorax and intracerebral hemorrhage). Due to the obstructive uropathy and anuria, a percutaneous nephrostomy was placed. However, death occurred within the first seven days after birth.

(4) Five infants were presented at a later time point with symptoms suggestive of PUV (e.g., difficulty urinating, weak stream of urine, enlarged bladder with a thick wall in the ultrasound). Here, primary urethral valve incision was carried out without prior urinary drainage.

Postnatal management of the 17 infants with cLUTO and a VAS indwelling included (Figure 4):

(1) Initial transurethral $(N=2)$ or suprapubic $(N=4)$ catheterization followed by either a valve incision $(N=$ $3)$ or a secondary vesicostomy $(N=3$; because a primary urethral valve incision was not possible due to the narrow urethral caliber).

(2) Early vesicostomy within the first 3 days of life $(N=4)$.

(3) Diversion by a tube through the existing VAS, a stent-instent-procedure $(N=5)$.

(4) Neonatal death within $48 \mathrm{~h}(N=2)$. In one patient a transurethral tube was inserted after birth. The second infant died in the first hour postnatal, hence no tube was inserted.

\section{Postnatal Urinary Diversion Through VAS}

In five neonates with VAS (Somatex ${ }^{\circledR}$ ) we secured urine drainage immediately after birth by placing a gastric tube $(N=1)$ or a 4.8 Fr JJ-stent $(N=4)$ through the lumen of the shunt (Figure 5). There were no patients with a Harrison fetal bladder stent in our cohort.

Within this group, the stent-in-stent diversion was later replaced by a suprapubic catheter in two cases: (1) Further treatment-including the placement of the suprapubic catheterwas continued in an external center. (2) A cystoscopy was performed at an age of 31 days, but an incision was not possible due to the narrow urethral caliber. After 37 days, the urethra was passed endoscopically without signs of hypoplasia and the valves were incised. In two other infants, the JJ-stent remained indwelling until the cystoscopy with definite urethral valve incision (for 55 days and 102 days, respectively). However, in one patient, the urethra was too narrow for intervention after 55 days. 


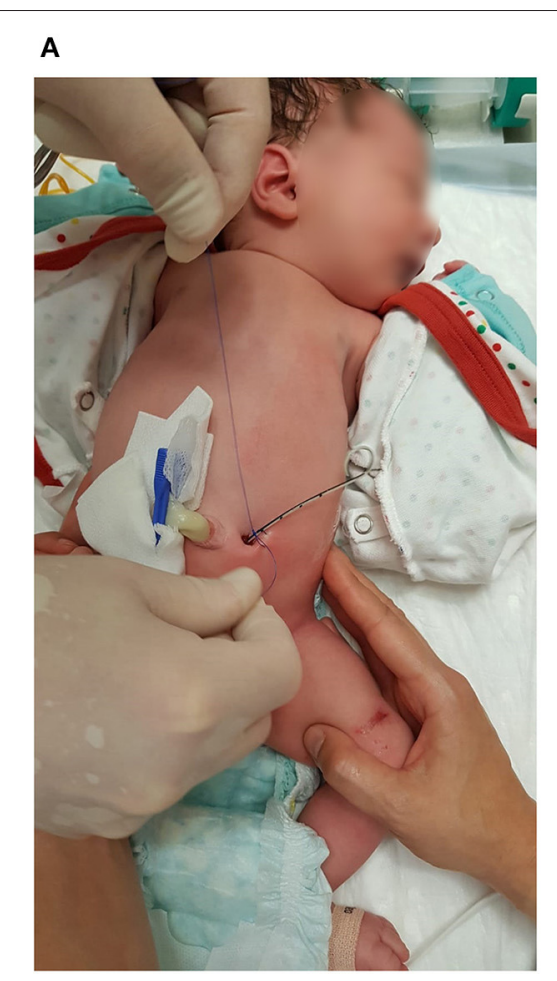

B

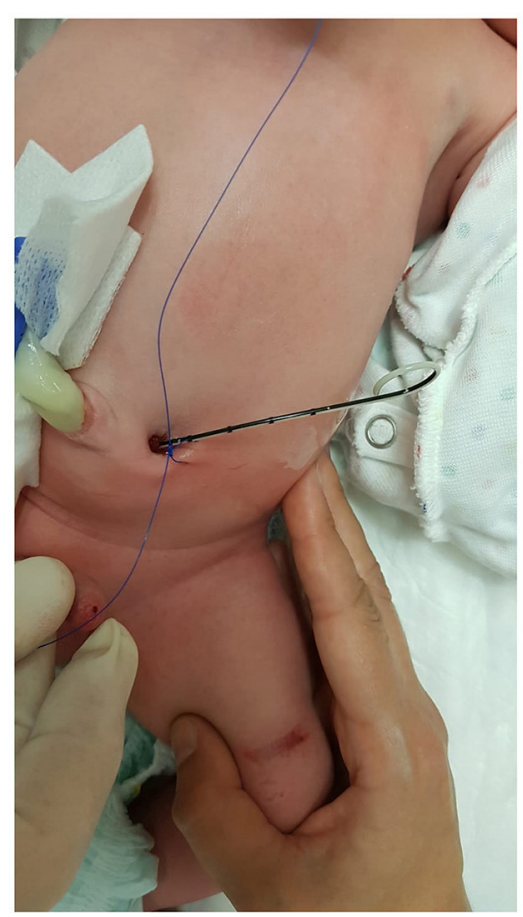

C

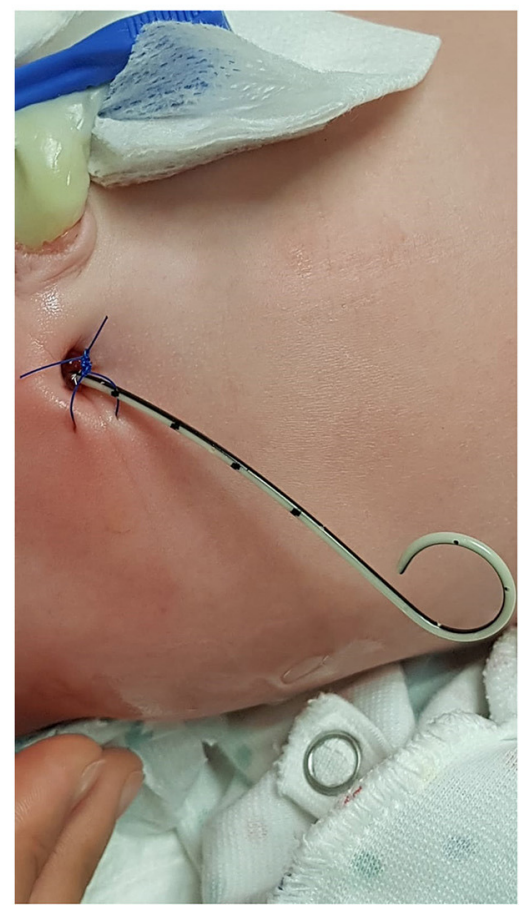

FIGURE 5 | Stent-in-stent scheme with a $4.8 \mathrm{Fr}$ JJ-catheter. The JJ-stent is fixed with a double knot on the skin surface close to the shunt exit site.

As a result, we suspected a urethral hypoplasia and performed a Blocksom vesicostomy. In another patient, urine drainage was secured by a JJ-stent but he died within seven days due to life-limiting comorbidities (urorectal septum malformation and complex congenital cardiac anomaly including a double outlet ventricle).

\section{DISCUSSION}

Up to $1 / 3$ of patients with cLUTO require renal replacement therapy in the long-term (5-7). Since 1983, an increasing number of affected fetuses treated with vesicoamniotic shunting has been reported (9). VAS are typically placed early, between the 12 th and the 14th week of gestation. This not only improves the survival rate of fetuses with oligo- or anhydramnios and associated pulmonary hypoplasia (11), but could also enhance the perinatal and the long-term renal function. Interventions performed at advanced stages in pregnancy are mainly beneficial for the fetal lung development, but not for the renal function. The PLUTO-trail - the only prospective, randomized trail analyzing the potential benefits of VAS - showed no difference between the two groups concerning the long-term renal function (12). In 2019, the latest meta-analysis from Chen et al. which included 13 studies analyzing the outcomes and prognostic factors associated with fetal megacystis showed, that intrauterine intervention did not significantly improve prognosis and survival rate (13).
The main challenge of VAS is to identify fetuses which will benefit from antenatal treatment and need intervention. First, there is a strong association between megacystis and chromosomal or structural abnormalities which further impair the prognosis (14). In $90 \%$ of the cases with a normal karyotype, megacystis $(7-15 \mathrm{~mm})$ resolves spontaneously before the 20th gestational week. On the contrary, spontaneous resolution is unlikely in neonates with a bladder length $>12 \mathrm{~mm}$ before the 18 th week or $>15 \mathrm{~mm}$ at the 20th week of gestation (15-17). In 2019, Fontanella et al. published a prognostic score to predict the severity of cLUTO using antenatal diagnostics. They stated that gestational age at the occurrence of oligo- or anhydramnios as well as urinary bladder volume at diagnosis can predict morbidity and mortality with a sensitivity of $\sim 78 \%$. Although this scoring system was developed in the Netherlands, it is mainly used in the USA (at the moment).

Since the beginning of the $21^{\text {st }}$ century, the role of fetal urine biochemistry in identifying candidates for in-utero intervention and predicting fetal outcome has been discussed (18). A sodium level $<100 \mathrm{mmol} / \mathrm{l}$, a chloride value $<90 \mathrm{mmol} / \mathrm{l}$ and an osmolarity $<200 \mathrm{mOsm} / \mathrm{l}$ in three consecutive fetal urine samples gained on three different days seem to indicate a better prognosis (19). Urinary biomarkers such as $\beta$-2-microglobline, sodium, chloride and calcium in urine samples before the 23rd week of gestation may be helpful to identify candidates for fetal therapy (20). Buffin-Meyer et al. could demonstrate in their most recent study, that the tested fetal urine peptide signature (12PUV) predicted postnatal renal outcome for suspected PUV with an 
AUC of 0.89 and an accuracy of $84 \%$ (21). Multi-center studies are necessary to confirm these findings.

Currently, European reference networks for rare urological disorders (ERN eUROGEN) and rare kidney diseases (ERN ERKNet) support such a study to generate reliable data (22). The status of amnion fluid, the renal ultrasound as well as the fetal urine biochemistry could be helpful in counseling prospective parents. The desirable aim for the future is to reliably predict, who would benefit from an early fetal intervention.

Nevertheless, after the baby is born pediatric urologists have to tend to the "surgical" management. At the moment, there are several shunts commercially available and more are being developed $(23,24)$. Some of the VAS, like the Somatec Shunt ${ }^{\circledR}$, have a larger lumen than the Rocket and Harrison shunt. As most shunts are placed at an early stage of pregnancy, urinary flow is diverted from the fetal urethra. Hypothetically, this could lead to a delayed development of the urethra causing further hypoplasia. In these cases, a transurethral catheterization is not possible. However, this is still a theory based on our own experience without any evidence.

The infants are mostly premature and/or small for gestation, hence they have an increased risk from general anesthesia. In the first 2 years (of our experience in postnatal treatment), we removed the inserted stents in general anesthesia, performed a temporary, incontinent Blocksom vesicostomy (25) and let the neonate grow, until a definitive treatment could be performed. However, performing surgery in this early stage of life can lead to a prolonged duration of ventilation and intensive care unit stay, as some of the newborns suffer from additional severe comorbidities or malformations.

In case of a VAS with a lumen surrounded by nitinol/metal, we placed a gastric tube (3-5 Fr) through the stent into the bladder to secure urine drainage. Since this procedure was successful and acute postnatal surgery could be avoided, we applied the stentin-stent scheme (4.8 Fr JJ-catheter) in the following four cases. Later, these catheters or stents can be used to perform a VCUG, avoiding any manipulation through the hypoplastic urethra.

\section{REFERENCES}

1. Malin G, Tonks AM, Morris RK, Gardosi J, Kilby MD. Congenital lower urinary tract obstruction: a population-based epidemiological study. BJOG. (2012) 119:1455-64. doi: 10.1111/j.1471-0528.2012.03476.x

2. Berte N, Vrillon I, Larmure O, Gomola V, Ayav C, Mazeaud C, et al. Long-term renal outcome in infants with congenital lower urinary tract obstruction. Prog Urol. (2018) 28:596-602. doi: 10.1016/j.purol.2018.06.005

3. Johnson MP, Danzer E, Koh J, Polzin W, Harman C, O'Shaughnessy R, et al. Natural history of fetal lower urinary tract obstruction with normal amniotic fluid volume at initial diagnosis. Fetal Diagn Ther. (2017) 44:10-7. doi: 10.1159/000478011

4. Ruano R, Dunn T, Braun MC, Angelo JR, Safdar A. Lower urinary tract obstruction: fetal intervention based on prenatal staging. Pediatr Nephrol. (2017) 32:1871-8. doi: 10.1007/s00467-017-3593-8

5. Heikkil J, Holmberg C, Kyllonen L, Rintala R, Taskinen S. Long-term risk of end stage renal disease in patients with posterior urethral valves. J Urol. (2011) 186:2392-6. doi: 10.1016/j.juro.2011.07.109

6. Smith GH, Canning DA, Schulman SL, Snyder HM, 3rd, Duckett JW. The long-term outcome of posterior urethral valves treated
Securing urine drainage in this manner allows the definitive treatment to be postponed until the early neonatal period is over and the infant is more stable. Moreover, the time gained could be used for more detailed diagnostics and further counseling of the parents.

\section{CONCLUSION}

Pediatric urologists are challenged with the management of vesicoamniotic stents in cLUTO patients after birth. In our opinion, having a secure urine drainage through the VAS offers the possibility to wait until the mostly premature infants have stabilized and grown. Hereby, the risk of general anesthesia is reduced and the urethral caliber can increase to allow cystoscopy. Hence, transurethral manipulation or a vesicostomy can be avoided.

\section{DATA AVAILABILITY STATEMENT}

The raw data supporting the conclusions of this article will be made available by the authors, without undue reservation.

\section{ETHICS STATEMENT}

The studies involving human participants were reviewed and approved by (Ethical Approval No. 2020-873R). Written informed consent to participate in this study was provided by the participants' legal guardian/next of kin.

\section{AUTHOR CONTRIBUTIONS}

MJ: collection of the data, analysis, wrote part the manuscript, and creation of Figure 1. MJ and NY: collection of the data, analysis, wrote part of the manuscript, and supervision of the results. RS: had the idea, wrote part of the manuscript, and supervision. All authors contributed to the article and approved the submitted version.

with primary valve ablation and observation. J Urol. (1996) 155:1730-4. doi: 10.1016/S0022-5347(01)66186-X

7. Farrugia MK. Fetal bladder outlet obstruction: embryopathology, in utero intervention and outcome. J Pediatr Urol. (2016) 12:296-303. doi: 10.1016/j.jpurol.2016.05.047

8. Liao AW, Sebire NJ, Geerts L, Cicero S, Nicolaides KH. Megacystis at 10-14 weeks of gestation: chromosomal defects and outcome according to bladder length. Ultrasound Obstet Gynecol. (2003) 21:338-41. doi: 10.1002/uog.81

9. Manning FA, Harman CR, Lange IR, Brown R, Decter A, MacDonald N. Antepartum chronic fetal vesicoamniotic shunts for obstructive uropathy: a report of two cases. Am J Obstet Gynecol. (1983) 145:819-22. doi: 10.1016/0002-9378(83)90685-3

10. Fontanella F, Duin L, Adama van Scheltema PN, Cohen-Overbeek TE, Pajkrt E, Bekker $\mathrm{M}$, et al. Antenatal workup of early megacystis and selection of candidates for fetal therapy. Fetal Diagn Ther. (2019) 45:15561. doi: $10.1159 / 000488282$

11. Strizek B, Gottschalk I, Recker F, Weber E, Flock A, Gembruch U, et al. Vesicoamniotic shunting for fetal megacystis in the first trimester with a Somatex $([\mathrm{R}])$ intrauterine shunt. Arch Gynecol Obstet. (2020) 302:13340. doi: 10.1007/s00404-020-05598-z 
12. Morris RK, Malin GL, Quinlan-Jones E, Middleton LJ, Hemming K, Burke D, et al. Percutaneous vesicoamniotic shunting versus conservative management for fetal lower urinary tract obstruction (PLUTO): a randomised trial. Lancet. (2013) 382:1496-506. doi: 10.1016/S0140-6736(13)60992-7

13. Chen L, Guan J, Gu H, Zhang M. Outcomes in fetuses diagnosed with megacystis: systematic review and meta-analysis. Eur J Obstet Gynecol Reprod Biol. (2019) 233:120-6. doi: 10.1016/j.ejogrb.2018. 12.007

14. Debska M, Kretowicz P, Oledzka A, Gastol P, Dangel J, Swiatkowska-Freund $\mathrm{M}$, et al. Early vesico-amniotic shunting - does it change the prognosis in fetal lower urinary tract obstruction diagnosed in the first trimester? Ginekol Pol. (2017) 88:486-91. doi: 10.5603/GP.a2017.0089

15. Cheung KW, Morris RK, Kilby MD. Congenital urinary tract obstruction. Best Pract Res Clin Obstet Gynaecol. (2019) 58:7892. doi: 10.1016/j.bpobgyn.2019.01.003

16. Fontanella F, Duin L, Adama van Scheltema PN, Cohen-Overbeek TE, Pajkrt E, Bekker M, et al. Fetal megacystis: prediction of spontaneous resolution and outcome. Ultrasound Obstet Gynecol. (2017) 50:45863. doi: 10.1002/uog.17422

17. Kagan KO, Staboulidou I, Syngelaki A, Cruz J, Nicolaides KH. The 11-13week scan: diagnosis and outcome of holoprosencephaly, exomphalos and megacystis. Ultrasound Obstet Gynecol. (2010) 36:10-4. doi: 10.1002/uog.7646

18. Fontanella F, van Scheltema PNA, Duin L, Cohen-Overbeek TE, Pajkrt E, Bekker MN, et al. Antenatal staging of congenital lower urinary tract obstruction. Ultrasound Obstet Gynecol. (2019) 53:520-4. doi: 10.1002/uog.19172

19. Freedman AL, Johnson MP, Gonzalez R. Fetal therapy for obstructive uropathy: past, present.future? Pediatr Nephrol. (2000) 14:167-76. doi: 10.1007/s004670050035

20. Abdennadher W, Chalouhi G, Dreux S, Rosenblatt J, Favre R, Guimiot F, et al. Fetal urine biochemistry at 13-23 weeks of gestation in lower urinary tract obstruction: criteria for in-utero treatment. Ultrasound Obstet Gynecol. (2015) 46:306-11. doi: 10.1002/uog.14734

21. Buffin-Meyer B, Tkaczyk M, Stanczyk M, Breuil B, Siwy J, Szaflik K, et al. A single-center study to evaluate the efficacy of a fetal urine peptide signature predicting postnatal renal outcome in fetuses with posterior urethral valves. Pediatr Nephrol. (2020) 35:469-75. doi: 10.1007/s00467-01904390-9

22. Buffin-Meyer B, Klein J, van der Zanden LFM, Levtchenko E, Moulos P, Lounis $\mathrm{N}$, et al. The ANTENATAL multicentre study to predict postnatal renal outcome in fetuses with posterior urethral valves: objectives and design. Clin Kidney J. (2020) 13:371-9. doi: 10.1093/ckj/ sfz107

23. Kurtz MP, Koh CJ, Jamail GA, Sangi-Haghpeykar H, Shamshirsaz AA, Espinoza J, et al. Factors associated with fetal shunt dislodgement in lower urinary tract obstruction. Prenat Diagn. (2016) 36:720-5. doi: $10.1002 /$ pd. 4850

24. Kurtz M. Improving the vesicoamniotic shunt for fetal lower urinary obstruction. Dialog Pediatr Urol. (2020) 2020:19-20.

25. Blocksom BH, Jr. Bladder pouch for prolonged tubeless cystostomy. J Urol. (1957) 78:398-401. doi: 10.1016/S0022-5347(17)66453-X

Conflict of Interest: The authors declare that the research was conducted in the absence of any commercial or financial relationships that could be construed as a potential conflict of interest.

Copyright (C) 2021 Jank, Stein and Younsi. This is an open-access article distributed under the terms of the Creative Commons Attribution License (CC BY). The use, distribution or reproduction in other forums is permitted, provided the original author(s) and the copyright owner(s) are credited and that the original publication in this journal is cited, in accordance with accepted academic practice. No use, distribution or reproduction is permitted which does not comply with these terms. 